\title{
Inflatable multichannel rectal applicator for adaptive image-guided endoluminal high-dose-rate rectal brachytherapy: design, dosimetric characteristics, and first clinical experiences
}

\author{
Maximilian P. Schmid, MD', Daniel Berger, PhD', Martin Heilmann, MD', Jessica Bör', Bernd Wisgrill, MSc', \\ Ursula Azizi-Semrad, MD', Nicole Nesvacil, PhD', Prof., Richard Pötter, MD'1,2, Christian Kirisits, Assoc. Prof., DScl,2, \\ Rainer Schmid, MDl \\ 'Department of Radiation Oncology, Comprehensive Cancer Center, ${ }^{2}$ Christian Doppler Laboratory for Medical Radiation Research \\ for Radiation Oncology, Medical University of Vienna, Vienna, Austria
}

\begin{abstract}
Purpose: To investigate the dosimetric results and first clinical experiences with a new designed balloon applicator with adjustable catheters for endoluminal brachytherapy for patients with locally advanced rectal cancer not undergoing surgery.

Material and methods: The applicator consists of an inflatable rectal balloon with six attached Foley catheters used as guidance for the inserted brachytherapy plastic needles. The construction of the applicator and the dosimetric profile in terms of representative dose points in $0,2,5,10 \mathrm{~mm}$ ipsilaterally and in $0 \mathrm{~mm}$ contralaterally are described. The first clinical outcomes in three patients are reported.

Results: For all three patients, a reproducible dose gradient was achieved. The surface dose on the target side was $204 \pm 19 \%$ of the normalized dose in $5 \mathrm{~mm}(100 \%)$ tissue depth, and $143 \pm 8 \%$ in $2 \mathrm{~mm}$ and $64 \pm 3 \%$ in $10 \mathrm{~mm}$ tissue depth, while the surface dose on the contra-lateral side was $20 \pm 8 \%$. After radiochemotherapy with 50 Gy external beam radiotherapy and concomitant administration of capecitabine, a HDR brachytherapy boost in 2-3 fractions of 7-10 Gy each was delivered. All patients achieved a clinical complete response 3 month after the treatment, and no major toxicity was observed.

Conclusion: The use of the applicator was clinically feasible, and resulted in a stable and reproducible dose distribution. First clinical results are promising.

J Contemp Brachytherapy 2017; 9, 4: 359-363 DOI: https://doi.org/10.5114/jcb.2017.69335
\end{abstract}

Key words: brachytherapy, radiotherapy, rectal cancer.

\section{Purpose}

Conservative treatment of locally advanced rectal cancer is currently receiving an increasing investigation, as surgery may significantly impair both short-term and long-term quality of life results. Whereas for T1 and T2 cancers, excellent results can be achieved using contact X-ray radiotherapy alone with $90 \%$ local tumor control rates [1], only $12-20 \%$ complete remissions are observed in more advanced tumors after standard preoperative radiochemotherapy with 45-50 Gy [2]. However, in these well-responding patients, local tumor control and cancerspecific survival do not appear to be inferior to patients undergoing preoperative radiochemotherapy and surgery [3]. To increase the response to radiochemotherapy, a local boost can be considered. A recent dose-response analysis revealed that a dose of 92 Gy would be required for a $50 \%$ tumor control rate [4]. Besides contact X-ray radiotherapy, high-dose-rate brachytherapy (HDR-BT) has been described as a boost technique $[5,6,7]$. The steep dose gradient of HDR-BT qualifies for localized, highly focused treatments of small volumes. However, an appropriately focused applicator system with asymmetrical dose distribution is required to achieve a therapeutic benefit with this dosimetric profile. In the following, we describe the design, technical and dosimetric characteristics of an inhouse developed applicator allowing asymmetrical dose distribution, we report on the feasibility of its use, and present some preliminary clinical results.
Address for correspondence: Maximilian P. Schmid, MD, Department of Radiation Oncology, Comprehensive Cancer Center, Medical University of Vienna, General Hospital of Vienna, Währinger Gürtel 18-20, A-1090 Vienna, Austria, phone: +43 14040026920, fax: +43 14040026930, $\bowtie$ e-mail: maximilian.schmid@akhwien.at
Received: 09.03 .2017 Accepted: 06.07.2017 Published: 30.08 .2017 


\section{Material and methods}

\section{Patients and treatment}

Three patients with locally advanced rectal cancer were treated in 2014 and 2015 at the Department of Radiation Oncology of the Medical University of Vienna with primary radiochemotherapy and image-guided brachytherapy. Patient 1 was 65 years old with a T2 N1 adenocarcinoma of the distal rectum. Patient 2 was 82 years old and had a T3 N0 adenocarcinoma of the distal rectum. Patient 3 was 86 years old with a T3 N1 adenocarcinoma of the distal rectum. All patients were treated with 3D conformal external beam radiotherapy (EBRT) up to 50 Gy with 2 Gy per fraction. Target volume delineation for EBRT was based on the recommendations by Roels et al. [8]. Concomitant chemotherapy with capecitabine $1,650 \mathrm{mg} / \mathrm{m}^{2}$ was administered daily during EBRT. Patient 1 was treated initially in preoperative intent, but the patient then refused surgery, whereas patients 2 and 3 were considered upfront as medically unfit for surgery. Figure 1 shows the local tumor extension on T2-weighted magnetic resonance imaging (MRI) for patient 3: (A) before radiochemotherapy, and (B) at week 3 during radiochemotherapy. After radiochemotherapy, all three patients received an endorectal HDR-BT boost. The CTV at brachytherapy was defined based on the tumor extension at diagnosis, but adapted according to the thickness of the rectal wall at the time of brachytherapy.

\section{Applicator}

The applicator consists of an inflatable endorectal balloon (RectalPro 75, QLRAD Inc, Miami, Florida, USA) and six attached Foley catheters with 14 F diameter (Folysil, Coloplast, Humlebaek, Denmark). Shortened front ends of the Foley catheters with a length of $20 \mathrm{~cm}$ are fixed to each other as well as to the endorectal balloon. They are fixed at the tip of the catheters (in the axis of the endorectal balloon through the existing holes) and at the level of the buckling of the endorectal balloon. $24 \mathrm{~cm}$ plastic needles (OncoSmart ProGuide, Elekta, Stockholm, Sweden) with $6 \mathrm{~F}$ diameter are inserted into the lumen of each Foley catheter and are used as the brachytherapy applicators. The endorectal balloon can be inflated up to $60 \mathrm{ml}$, which results in 4.0-4.5 cm balloon diameter. The Foley catheters, with the brachytherapy applicators inside, are attached at the less inflatable side of the balloon (treatment area), and the more inflatable side of the balloon is used as a spacer towards the contralateral non-involved rectal wall. This construction results in an asymmetric arrangement of the brachytherapy source channels, which allows delivering a high dose to the target area while keeping the dose relatively low on the contra-lateral side of the rectum. Figure 2 shows the applicator in two different views.

\section{Applicator commissioning, reconstruction, and treatment planning}

Before clinical use, the fully mounted applicator was commissioned using a phantom computed tomography (CT) study. Therefore, autoradiography of the applicator was performed to define the offset (distance from the visible applicator tips to the first dwell positions) and the appropriate indexer length for the afterloader. A dummy wire was inserted into each plastic needle to visualize the first source position. When the needles were properly inserted into the Foley catheters, the offset according to our equipment and configuration was $8 \mathrm{~mm}$ when an indexer length of 1,407 $\mathrm{mm}$ for each channel was selected.

For the reconstruction of the applicator, CT images with a slice thickness of $2 \mathrm{~mm}$ were used and each catheter was digitized. The average distance between catheters was $9.0 \pm 0.4 \mathrm{~mm}$ with an active length of $4 \pm 1 \mathrm{~cm}$. The dose was normalized at $5 \mathrm{~mm}$ tissue depth, while
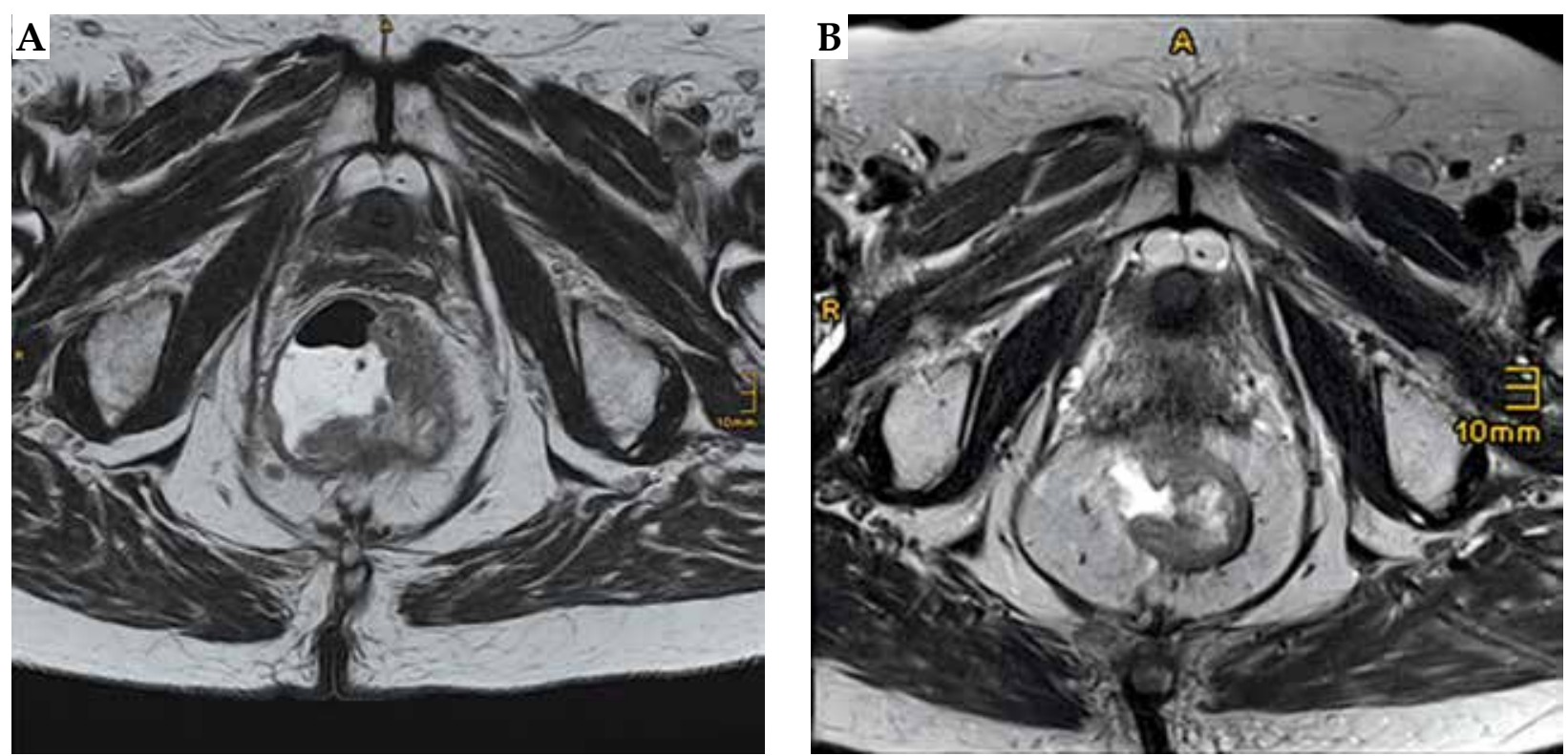

Fig. 1. T2-weighted magnetic resonance imaging (MRI) of patient 3 (T3 N1) (A) before external beam radiotherapy and chemotherapy, and (B) in week 3 during radiochemotherapy 

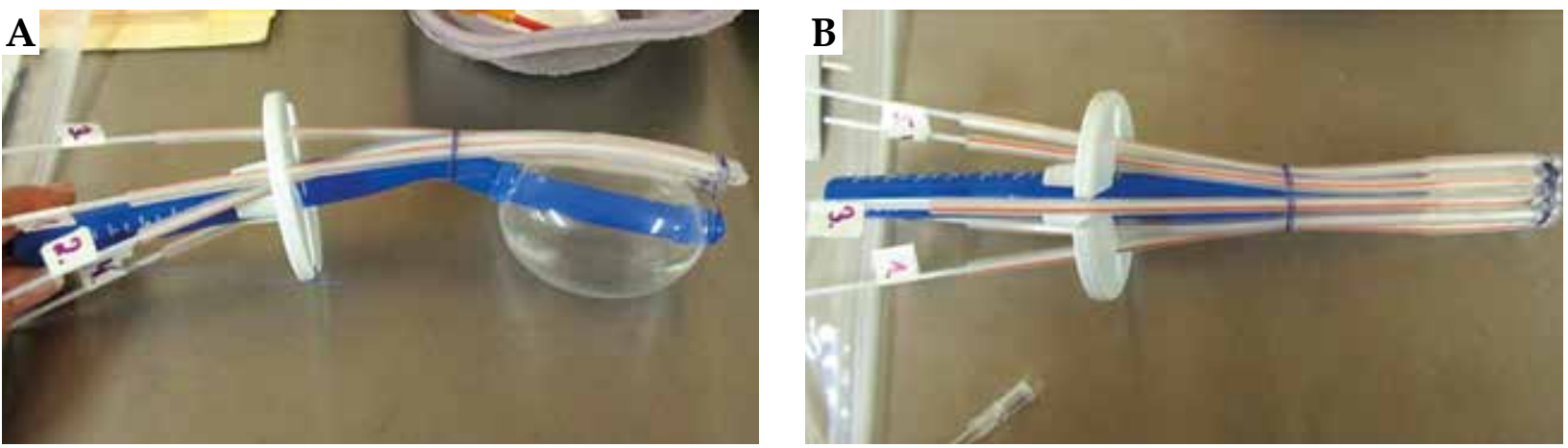

Fig. 2. Two views of the inflatable multi-channel rectal applicator for rectal brachytherapy (A) with inflated balloon, and (B) the configuration of the channels inside the shortened Foley catheters

additional dose points were defined at $0 \mathrm{~mm}$ (surface of the applicator $2 \mathrm{~mm}$ from the source), $2 \mathrm{~mm}, 5 \mathrm{~mm}$, and $10 \mathrm{~mm}$ on the target side and on the applicator surface contra-laterally. Geometric optimization with a minor manual adjustment of dwell times was mainly performed to shape the dose according to the geometry and patients' target volumes.
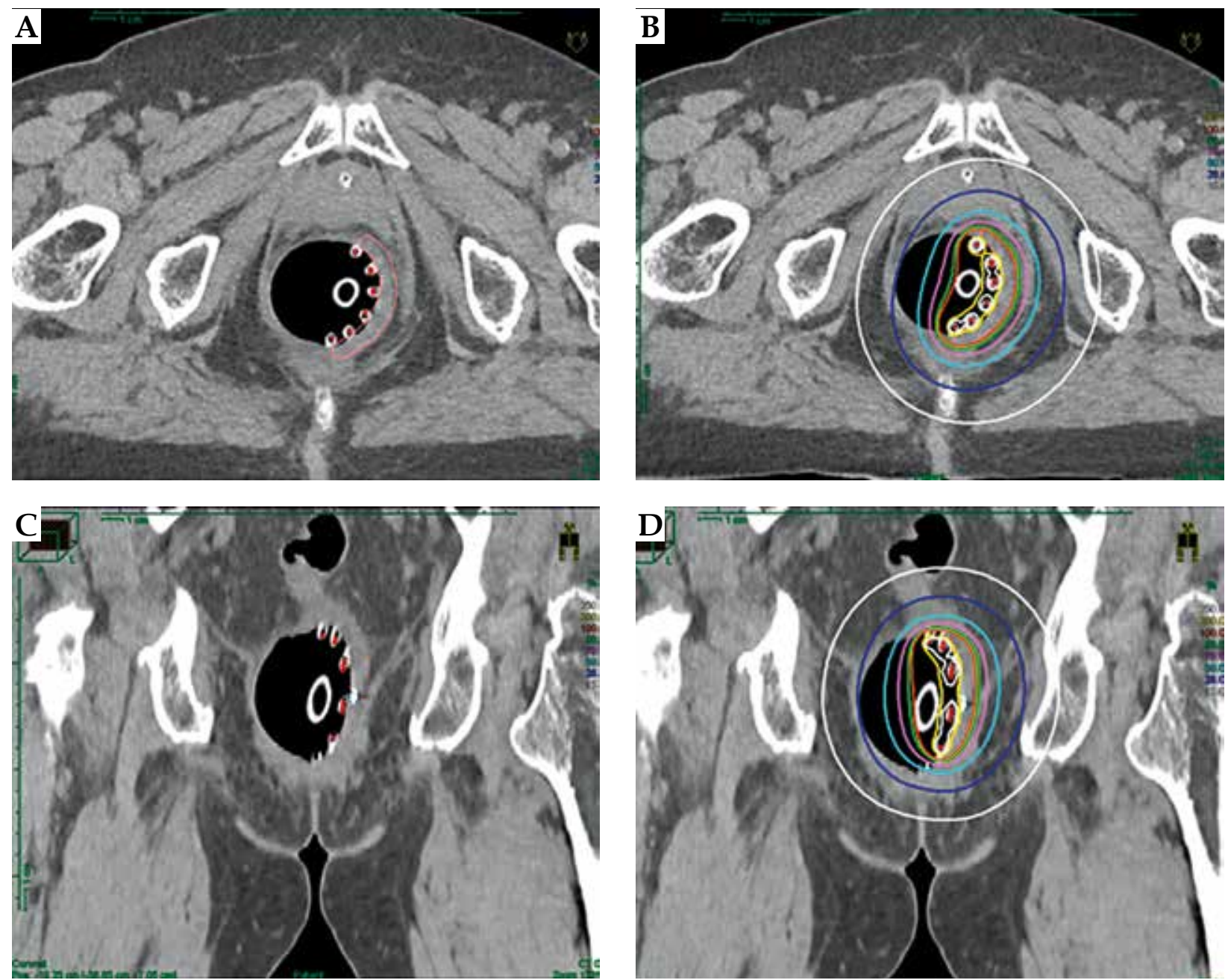

Fig. 3. Computed tomography images of patient 3 with inflated applicator in situ showing the dose distribution in transverse (A and $\mathbf{B})$ and coronal view $(\mathbf{C}$ and $\mathbf{D})$
In addition, a CTV encompassing the area of tumor extension at time of diagnosis and the thickness of the rectal wall at the time of brachytherapy was retrospectively delineated for dose-volume-histogram evaluation (Figure 3). Oncentra Brachytherapy 4.5.2 (Elekta, Stockholm, Sweden) was used for treatment planning. 


\section{Results}

\section{Clinical results}

Patient 1 and patient 2 achieved complete endoscopic remission after EBRT and chemotherapy, whereas rectoscopy in patient 3 revealed residual tumor (Figure 4). Gross target volume (GTV) at diagnosis and clinical target volume (CTV) at brachytherapy were, respectively, $2.7 \mathrm{~cm}^{3}$ and $2.2 \mathrm{~cm}^{3}$ (45\% involvement of the circumference of the rectum) in patient $1,12.8 \mathrm{~cm}^{3}$ and $2.8 \mathrm{~cm}^{3}$ (45\% involvement of the circumference) in patient 2 , and $42.7 \mathrm{~cm}^{3}$ and $18.0 \mathrm{~cm}^{3}$ (60\% involvement of circumference) in patient 3. Positioning of the applicator was well-tolerated by the patients who did not require any anesthesia. The fully mounted deflated applicator was inserted into the rectum according to the required rotation and insertion depth, and inflated up to $40-60 \mathrm{ml}$ based on the patient's tolerance. By inflating the balloon, the catheters were pressed against the rectal wall. The position of the applicator was checked with CT imaging and adjusted if necessary. After CT-based treatment planning with the inflated applicator in situ (see above), the treatment was applied without any complications and the applicator was removed directly after. The overall procedure including rectoscopy was performed within approximately 1-2 hours as an out-patient treatment.

All three patients showed complete clinical remission after treatment. Currently, all the patients are alive with no evidence of disease. Patient 1 developed a superficial ulceration causing pain one year after treatment, which was treated conservatively. Patient 3 developed minor rectal bleedings. None of the patients required colostomy after radiotherapy.

\section{Dosimetric results}

For all three patients, a similar dose gradient was achieved. The surface dose on the target side was $204 \pm$ $19 \%$ of the normalized dose at $5 \mathrm{~mm}(100 \%)$ tissue depth, $143 \pm 8 \%$ at $2 \mathrm{~mm}$, and $64 \pm 3 \%$ at $10 \mathrm{~mm}$, while the surface dose on the contra-lateral side was only a tenth of the dose to the target side, resulting in $20 \pm 8 \%$. The active length was $3 \mathrm{~cm}$ for patient $1,4 \mathrm{~cm}$ for patient 2 , and $5 \mathrm{~cm}$ for patient 3 , with resulting TRAKs (total reference air kerma) of $0.19 \mathrm{cGy} / 1 \mathrm{~m}, 0.27 \mathrm{cGy} / 1 \mathrm{~m}$, and $0.39 \mathrm{cGy} / 1 \mathrm{~m}$, respectively. While the dose prescriptions for patient 1 and patient 2 were 7 Gy at $5 \mathrm{~mm}$ for 2 and 3 fractions, respectively, patient 3 was treated with $10 \mathrm{~Gy}$ at $5 \mathrm{~mm}$ for 3 fractions. The $\mathrm{D}_{90}$ doses of the retrospectively delineated CTV were $7.5 \mathrm{~Gy}\left(\mathrm{EQD}_{2}\right.$ - equivalent dose at $2 \mathrm{~Gy}), 12.2 \mathrm{~Gy}\left(\mathrm{EQD}_{2}\right)$, and $12.2 \mathrm{~Gy}\left(\mathrm{EQD}_{2}\right)$ per fraction for patients 1,2, and 3, respectively. In total (EBRT + all brachytherapy fractions), doses of $65 \mathrm{~Gy}\left(\mathrm{EQD}_{2}\right)$, 87 Gy $\left(\mathrm{EQD}_{2}\right)$, and $87 \mathrm{~Gy}\left(\mathrm{EQD}_{2}\right)$ were delivered to the $\mathrm{D}_{90}$ of the CTV of patients 1,2 , and 3 , respectively. Figure 3 shows the dose distribution of patient 3 in coronal and transverse views.

\section{Discussion}

Dose escalation to the tumor beyond the standard 4550 Gy radiochemotherapy can be considered in cases of conservative treatment of locally advanced rectal cancer. Data, mainly from Lyon/Nice and Clatterbridge, indicate high local tumor control and survival rates for advanced tumors if an additional boost is applied $[5,9,10]$. Contact X-rays or brachytherapy currently appear to be the only effective and safe ways to reach the presumably required high doses of $>90$ Gy [4]. Here, we described our experiences with our own applicator for adaptive image-guided endorectal HDR-BT. The applicator was designed to provide a high dose to the target volume, while simultaneously sparing the non-involved circumference of the rectum. This was enabled by adding an inflatable balloon as a spacer to the guiding tubes for the plastic needles. This configuration provides a steep dose gradient in the target volume, and substantially reduces the dose to the contralateral side. Jakobsen et al. [11] reported the use of a $20 \mathrm{~mm}$ cylinder as an applicator including additional shields. With this approach, an asymmetric dose profile can be achieved as well - approximately $30 \%$ of the prescribed dose reaches the con-
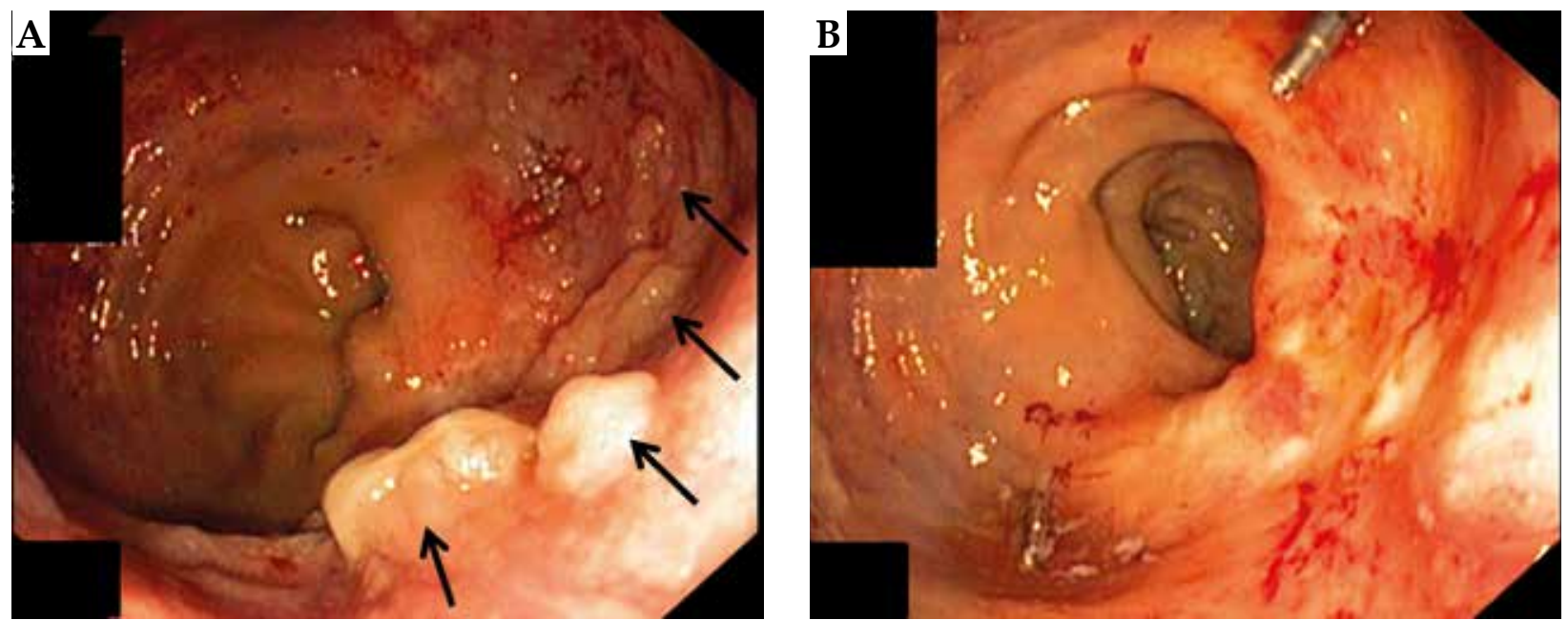

Fig. 4. Rectoscopy of patient 3: (A) before brachytherapy showing residual tumor (black arrows), and (B) three months after brachytherapy showing complete endoscopic remission (two computed tomography markers in situ) 
tralateral side behind the shields [12]. However, a cylinder only allows for limited adjustments according to the individual anatomy. The fixation of the cranio-caudal applicator position in relation to the target, and the additional need for an advanced dose calculation algorithm to include shielding material into the dose calculation, raises some practical challenges. While the Novi Sad applicator uses a similar principle as our applicator, with an inflatable balloon allowing for a better fixation and asymmetric dose distribution, our applicator appears to provide a better dose ratio between target and not-involved rectal circumference.

Our applicator could be easily inserted and was welltolerated by the patients. Image-guided treatment planning was performed with $\mathrm{CT}$, which allowed for further dose optimization. The dose distribution was reproducible in all three patients with minor variations. The first patient received a relatively low dose in two fractions of $7 \mathrm{~Gy}$ at $5 \mathrm{~mm}$ tissue depth, which was then increased to three fractions of $10 \mathrm{~Gy}$ at $5 \mathrm{~mm}$ tissue depth in the third patient. No major acute toxicity was observed. A comparison of the point dose parameters with the respective DVH-parameters shows (as previously reported, e.g. for cervical cancer [13]) substantial differences. Additional research will be necessary to clarify the clinical relevance of these differences.

Further dose escalation might be considered based on results from the published series. With contact X-rays, three fractions of 30 Gy surface dose are recommended as a boost for advanced disease [14], while the optimal dose schedule for HDR-BT is not clear. The Montreal group applies three fractions of $10 \mathrm{~Gy}$ at $10 \mathrm{~mm}$ from the applicator surface (after 50.4 Gy EBRT), whereas the HERBERT trial recommends three fractions of $7 \mathrm{~Gy}$ at $10 \mathrm{~mm}$ from the applicator surface (after 39 Gy EBRT) [15].

Both contact X-rays and HDR-BT offers certain advantages in localized targeted treatment. While treatment with contact $X$-rays has biological and dosimetric advantages, and allows for direct sight of the tumor ("eye-guided treatment"), brachytherapy opens up the possibility for three-dimensional image-guided treatment planning and dose optimization and reporting. This allows a higher degree of freedom for individualized dose prescription, respecting tissue depths and tumor volumes, including bulkier and more infiltrative residual rectal tumors than those applicable for contact X-rays [16].

\section{Conclusion}

The use of the applicator was clinically feasible, and resulted in a stable and reproducible dose distribution. First clinical results are promising.

\section{Conflict of interest}

The Department of Radiotherapy at the Medical University of Vienna received financial and/or equipment support for research and educational purposes from Elekta Brachytherapy, Elekta AB and Varian Medical Systems, Inc.

\section{References}

1. Gérard JP, Ayzac L, Coquard R et al. Endocavitary irradiation for early rectal carcinomas T1 (T2). A series of 101 patients treated with the Papillon's technique. Int J Radiat Oncol Biol Phys 1996; 34: 757-783.

2. Patel UB, Taylor F, Blomqvist L et al. Magnetic resonance imaging-detected tumor response for locally advanced rectal cancer predicts survival outcomes: MERCURY experience. J Clin Oncol 2011; 29: 3753-3760.

3. Renehan AG, Malcomson L, Emsley R et al. Watch-and-wait approach versus surgical resection after chemoradiotherapy for patients with rectal cancer (the OnCoRe project): a propensity-score matched cohort analysis. Lancet Oncol 2016; 17 : 174-183.

4. Jakobsen A, Ploen J, Vuong T et al. Dose-effect relationship in chemoradiotherapy for locally advanced rectal cancer: a randomized trial comparing two radiation doses. Int J Radiat Oncol Biol Phys 2012; 84: 949-954.

5. Sun Myint A, Lee CD, Snee AJ et al. High dose rate brachytherapy as a boost after preoperative chemoradiotherapy for more advanced tumors: the Clatterbridge experience. Clin Oncol (R coll Radiol) 2007; 19: 711-719.

6. Vuong T, Devic S. High-dose-rate pre-operative endorectal brachytherapy for patients with rectal cancer. J Contemp Brachytherapy 2015; 7: 183-188.

7. Devic S, Vuong T, Moftah B. Advantages of inflatable multichannel endorectal applicator in the neo-adjuvant treatment of patients with locally advanced rectal cancer with HDR brachytherapy. J Appl Clin Med Phys 2005; 6: 44-49.

8. Roels S, Duthoy W, Haustermans Ket al. Definition and delineation of the clinical target volume for rectal cancer. Int J Radiat Oncol Biol Phys 2006; 65: 1129-1142.

9. Gerard JP, Chapet O, Ramaioli A, Romestaing P. Long-term control of T2-T3 rectal adenocarcinoma with radiotherapy alone. Int I Radiat Oncol Biol Phys 2002; 54: 142-149.

10. Gerard JP, Frin AC, Doyen J et al. Organ preservation in rectal adenocarcinoma (T1) T2-T3 Nx M0. Historical overview of the Lyon Sud - Nice experience using contact X-ray brachytherapy and external beam radiotherapy for 120 patients. Acta Oncol 2015; 54: 545-551

11. Jakobsen A, Mortensen JP, Bisgaard C et al. Preoperative chemoradiation of locally advanced $\mathrm{T} 3$ rectal cancer combined with an endorectal boost. Int J Radiat Oncol Biol Phys 2006; 64: 461-465.

12. Poon E, Reniers B, Devic $S$ et al. Dosimetric characterization of a novel intracavitary mold applicator for 192Ir high dose rate endorectal brachytherapy treatment. Med Phys 2006; 33 : 4515-4526.

13. Tanderup K, Nielsen SK, Nyvang GB et al. From point A to the sculpted pear: MR image guidance significantly improves tumour dose and sparing of organs at risk in brachytherapy of cervical cancer. Radiother Oncol 2010; 94: 173-180.

14. Gerard JP, Chapet O, Nemoz C et al. Improved sphincter preservation in low rectal cancer with high-dose preoperative radiotherapy: the lyon R96-02 randomized trial. J Clin Oncol 2004; 22: 2404-2409.

15. Sun Myint A, Lee CD, Gerard JP. Rectal cancer. The GEC ESTRO Handbook of Brachytherapy. $2^{\text {nd }}$ ed. 2014.

16. Nout RA, Devic S, Niazi T et al. CT-based adaptive highdose-rate endorectal brachytherapy in the preoperative treatment of locally advanced rectal cancer: Technical and practical aspects. Brachytherapy 2016; 15: 477-484. 